\title{
Specificity of brain activity in response to acoustic stimulation
}

N.Almayev, O. Murasheva, Yu. Bessonova, A. Medincev, Institute of Psychology, Russian Academy of sciences

This work is devoted to the search of the mechanisms of sound's influence on human organism. Main feature of our approach is the isolated variation of one-two characteristics of sound while experimental control of all of the other.

METHOD Effect of the primitive rhythms (pulsations) on CNS was measured by EEG. Six stimuli (adapted from Almayev and Skorik, 2015) those varied both in tempo and loudness were administered quasi randomly to 26 persons (12 male, 14 female). Duration of active phase of all stimuli was $150 \mathrm{~ms}$, while pauses were 100, 500 and $1200 \mathrm{~ms}$, the levels of loudness $90 \mathrm{~dB}$ and $70 \mathrm{~dB}$, duration of whole stimulus $33 \mathrm{sec}$. Stimuli got numbers 1 (100 ms pause, $90 \mathrm{~dB}), 2$ (500 $\mathrm{ms}, 90 \mathrm{~dB}), 3(1200 \mathrm{~ms}, 90 \mathrm{~dB}), 4(100 \mathrm{~ms}$, $70 \mathrm{~dB}), 5(500 \mathrm{~ms}, 70 \mathrm{~dB})$ and $6(1200 \mathrm{~ms}$, $70 \mathrm{~dB}$ ). EEG was registered by Mitsar 202 encephalograph, according to 10-20 scheme, eyes closed. Only power of signals in six main EEG bands (not ERPs, nor coherence) was analyzed. Subjects also performed subjective estimation of the stimuli by ten Lickert-type scales: "Tension", "Irritation", "Approach", "Acceleration", "Concentration", "Desire to do", "Expectation", "Anxiety", "Interest". They also have done some psychological tests.

RESULTS

No differences between conditions comparable to within group differences were found in any band of EEG rhythms. The value of $F$ relations for the factors: tempo, loudness, tempo*loudness varied from 0,43 (the smallest) to 1,18 (the biggest), while that of Intercept from 17,25 (the smallest) to 54,33 (the biggest). This results contrasts with our previous findings (2016) when the stimuli were united into two sequences with

This work was supported by RFBR grant \# 16-06-00487 accelerating tempo and loudness of $70 \mathrm{~dB}$ and $90 \mathrm{~dB}$. Significant differences between the state of the rest and both of 3 minutes sequences were found in Delta, Theta, Alpha and Beta 2 bands. Differences between the two 3 minutes sequences were also observed at some of the leads within Alpha, Delta and Theta bands. Rough estimation of the stimulation time required for significant change in EEG power is therefore, several minutes. Although, $33 \mathrm{sec}$ sequences are not sufficient for significant between conditions changes some tendencies in the influence of the kind of stimuli on EEG rhythms were observed. The most prominent of them are detected in Theta band, especially in Fp1,Fp2, Fpz, F3, Fz, C3, Cz leads. Slowing of tempo tends to reduce the power of Theta rhythm in this leads. On the contrary for Alpha band there is tendency of power growth in P3, Pz, P4 leads with the slowing of tempo (see the charts). These findings correspond to wide spread views that Theta rhythm is connected to the scanning of environment while Alpha rhythm - to subcortical stimulation.

The need of several minutes long intensive stimulation in order to cause substantial differences in power of EEG activity contrasts to the time needed for subjective estimation of stimuli. Our recent studies (2018) show that only about $300 \mathrm{~ms}$ is sufficient for discrimination between major and minor chords by non musicians. The difference in hundreds of times points at substantial difference of the processes involved. Nevertheless, total of $\mathbf{2 7 1}$ significant $(p<0,05)$ correlations were found between subjective estimations of stimuli and power of EEG rhythms in different bands and different leads.
For example, stimulus 1 has ten correlations (from $r=0,39$ to $r=0,56$ ) in Gamma, Beta and Beta 2 bands eight of them with the scale "Approach" (leads F4, C4, T4, P4, O2, $\mathrm{Cz})$, one with "Interest" $(\mathrm{r}=0,56$; $\mathrm{O} 2)$ and one in Delta band with "Acceleration" ( $r=0,46 ; F 7)$. Remarkable affinity is observed between subjective scale, stimulus and EEG activity. Thus, subjective scale "Tension" has 12 correlations, 8 of them with stimulus 2 in Beta 2 band (from $r=-39$ to $r=-0,54$ ), leads $F p 1$, $\mathrm{Fpz}, \mathrm{P} 3, \mathrm{Pz}, \mathrm{P} 4, \mathrm{~T} 6, \mathrm{Oz}, \mathrm{O} 2$, and one in Alpha band $r=-0,53,(\mathrm{O} 2)$. The other three correlations of the "Tension" scale refer to stimuli 6 (Alpha, $r=0,69$; O2, Delta, $r=0,788$, $\mathrm{O} 2$ ) and 5 (Theta, $r=0,39 ; \mathrm{Fpz}$ ). "Anxiety" scale has 21 correlations, 20 of them with stimulus 2, 12 are in Alpha band (from $r=$ 0,37 to $r=-0,52$ ), electrodes Fp1, F7, F3, Fz, C3, T5, P3, Pz, T6, O1, Oz, O2, the other 8 lay in Delta, Theta and Beta 1 bands.
"Interest" scale has total of 48 correlations from which 34 are within stimulus 3 and 11 within stimulus 6 , the tempo of these stimuli is the same. The meaning of this correlations generally correspond to contemporary views on the nature of EEG bands, e.g. there were no positive correlations of Anxiety and Alpha rhythm, etc. Similar correlations were found as well between the subjects estimations of themselves on the base of temperamental questionnaire (Rusaloff) and power of EEG rhythms in the state of the rest, eyes closed.

Taking the above into consideration it is likely that subjective estimations may highlight the tendencies in EEG data.

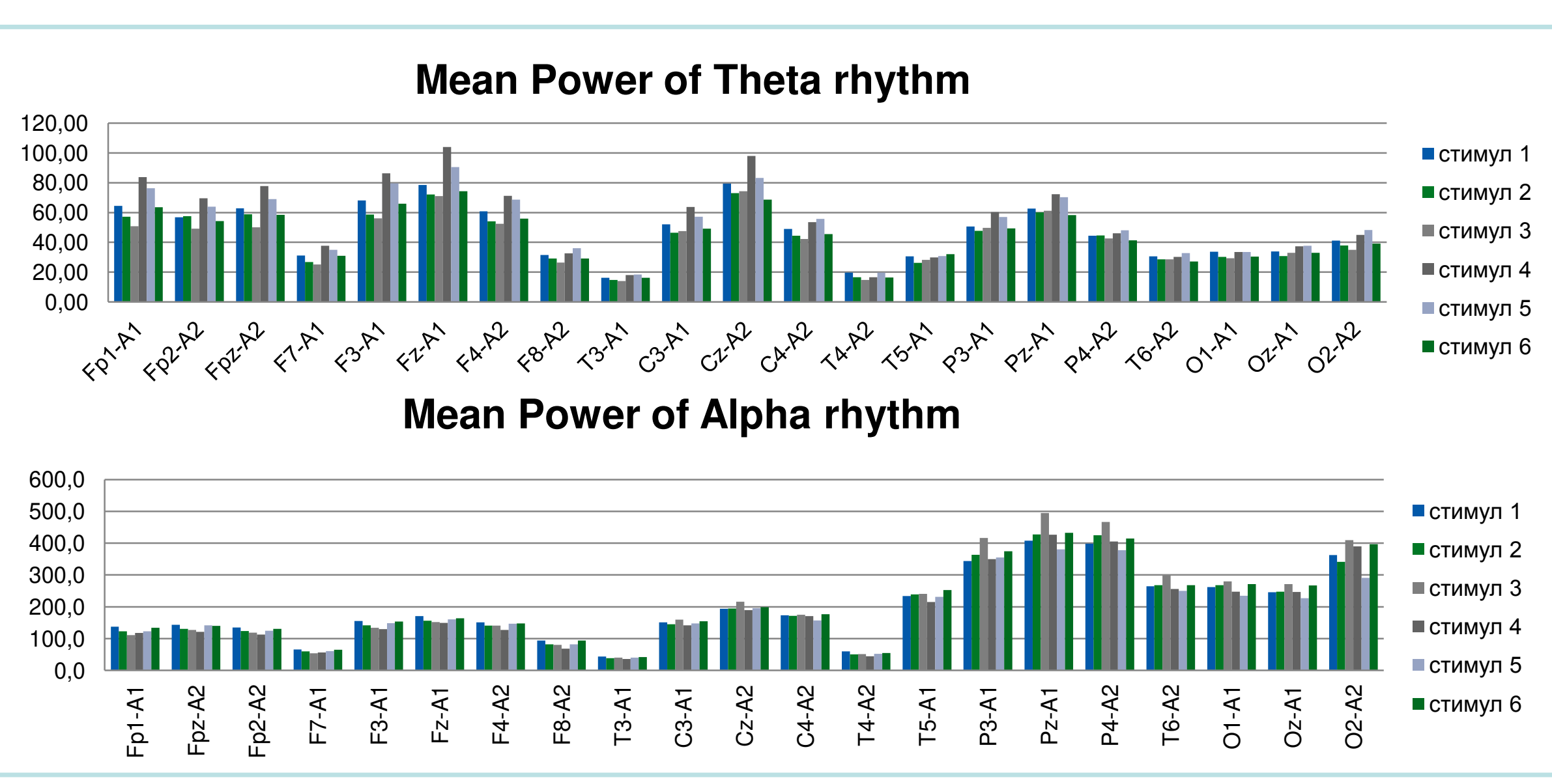

